\title{
BOUNDARY IDENTIFICATION FOR AN ELASTIC SOLID PARTLY IMMERSED IN A LIQUID
}

\author{
Dang Dinh Ang ${ }^{1}$ Nguyen Dung ${ }^{2}$ Dang Duc Trong ${ }^{1}$ \\ 1 University of Natural Sciences HoChiMinh City \\ ${ }^{2}$ Institute of Applied Mechanics, HoChiMinh City
}

\begin{abstract}
The authors consider a long elastic cylinder of constant section $\Omega$ partly immersed in a liquid of constant density. It is assumed that the body is in the state of plane strain and that $\Omega$ has a boundary consisting of two piecewise smooth arcs assumed to meet each other at two, and only two, points on a horizontal line. The upper arc $\Gamma$, which is exposed to air, is assumed known, while the lower arc $\gamma$, assumed to be totally immersed in a liquid of constant density, is unknown and is to be determined. Under the conditions that the displacements and surface stresses on a subarc of $\Gamma$ are known and that the lower arc $\gamma$ is subjected to a known constant hydrostatic pressure, the authors prove a uniqueness theorem and in the case of existence of a solution, show the existence of a sequence of regularized solutions converging to the exact solution.
\end{abstract}

\section{Introduction}

Consider a long elastic cylinder of constant (cross) section as shown in Fig. 1. Plane strain is assumed. The section is represented as the union of two piecewise smooth arcs $\Gamma$ and $\gamma$ having two, and only two, common points, these points lying on a horizontal line. The arc $\Gamma$ is assumed to be above $\gamma$ and to be exposed to air whereas $\gamma$ is completely immersed in a liquid of constant density. It is assumed that the elastic body is so light that $\gamma$ is near the surface of the liquid. Therefore we can safely assume, as a first approximation, that the hydrostatic pressure acting on $\gamma$ is

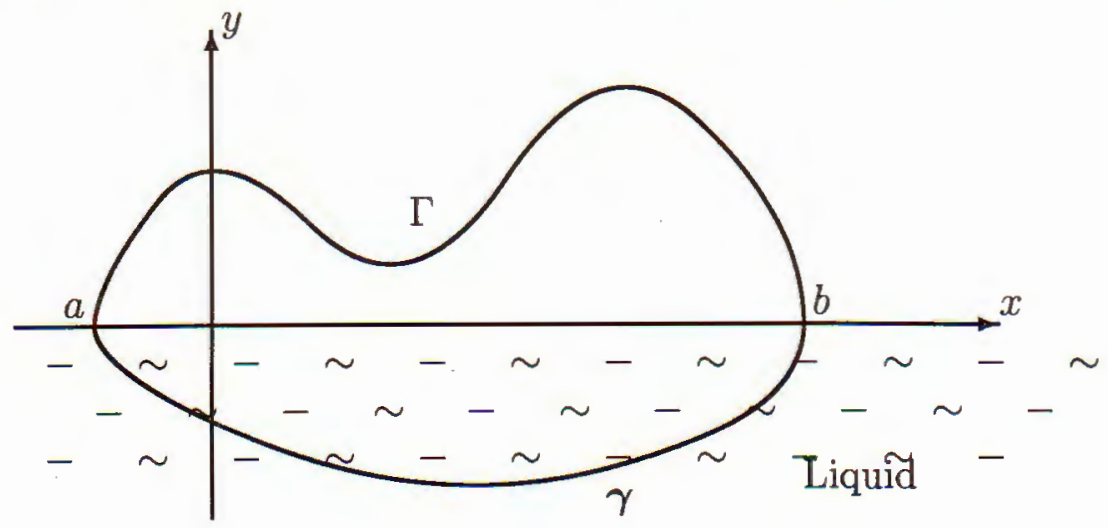

Fig. 1 
a (known) constant. The state of plane strain being assumed, the problem will be treated two-dimensionally, in $x, y$ and the equation of equilibrium will be on the domain $\Omega$, which is a section of the cylinder. It will be assumed that the surface displacements and surface stresses are specified on $\Gamma$ (see Fig. 1). Before proceeding further, we note that the following analysis, with some modifications, will go through if conditions on displacements and stresses are given on a subarc of $\Gamma$, however for simplicity in the presentation, we assume these conditions to be specified on the whole of $\Gamma$.

Under the foregoing conditions, we prove a uniqueness theorem, and in the case of existence of a solution, we show the existence of a sequence of approximate solutions converging to the exact solution.

\section{Mathematical Formulation}

Let the elastic body $\Omega$ be referred to a Cartesian system of coordinates $x, y$ as shown in Fig. 1. Using the notations of Timoshenko and Goodier [TG], we denote the displacements in the $x$-direction and the $y$-direction respectively by $u, v$ and let $\varepsilon_{x}, \varepsilon_{y}, \gamma_{x y} \sigma_{x}, \sigma_{y}, \tau_{x y}$ be the strain and stress components. Assuming plane strain, we have

$$
\left\{\begin{array}{l}
\varepsilon_{x}=\frac{\partial u}{\partial x}, \varepsilon_{y}=\frac{\partial v}{\partial y}, \gamma_{x y}=\frac{\partial u}{\partial y}+\frac{\partial v}{\partial x} \\
\tau_{x y}=G\left(\frac{\partial u}{\partial y}+\frac{\partial v}{\partial x}\right) \\
\sigma_{x}=\frac{E \nu}{(1+\nu)(1-2 \nu)}\left(\varepsilon_{x}+\varepsilon_{y}\right)+\frac{E}{1+\nu} \varepsilon_{x} \\
\sigma_{y}=\frac{E}{(1+\nu)(1-2 \nu)}\left(\varepsilon_{x}+\varepsilon_{y}\right)+\frac{E}{1+\nu} \varepsilon_{y}
\end{array}\right.
$$

where $E, \nu$ are positive constants satisfying

$$
E=2 G(1+\nu), \quad 0<\nu<\frac{1}{2} .
$$

With $X, Y$ denoting the body forces in $x$-, $y$-directions respectively, we have the following equilibrium equations (cf. [TG])

$$
\left\{\begin{array}{l}
\frac{\partial \sigma_{x}}{\partial x}+\frac{\partial \tau_{x y}}{\partial y}+X=0 \\
\frac{\partial \sigma_{y}}{\partial y}+\frac{\partial \tau_{x y}}{\partial x}+Y=0
\end{array}\right.
$$

On the known boundary $\Gamma$, we assume

$$
(u, v)=(f, g)
$$




$$
\left\{\begin{array}{l}
\ell \sigma_{x}+m \tau_{x y}=F \\
m \sigma_{y}+\ell \tau_{x y}=G
\end{array}\right.
$$

where $(\ell, m)$ is the outer unit normal vector to $\partial \Omega$ and $(F, G)$ are surface stresses. Note that if the known boundary $\Gamma$ is exposed to air then we can take $F$ and $G$ to be 0 . In the following, since this involves no extra cost, we shall take $F$ and $G$ to be rather arbitrary functions.

Now, $\gamma$ is assumed to be piecewise $C^{1}$. Accordingly, there exists a finite subset $\left\{y_{1}, \ldots, y_{k}\right\}$ of $\gamma$ such that

$$
\gamma_{*} \equiv \gamma \backslash\left\{y_{1}, \ldots, y_{k}\right\}
$$

is a finite disjoint union of $C^{1}$-arcs.

The boundary $\gamma$ is assumed to be under constant hydrostatic pressure, i.e., there is a constant $p$ such that

$$
\left\{\begin{array}{l}
\ell \sigma_{x}+m \tau_{x y}=p \ell \\
m \sigma_{y}+\ell \tau_{x y}=p m
\end{array} \quad \text { on } \gamma_{*} .\right.
$$

\section{Uniqueness and Approximation}

Theorem 1 (Uniqueness theorem). If the unknown boundary $\gamma$ of $\Omega$ is a finite disjoint union of piecewise $C^{1}$-Jordan arcs and if we have either or

(a) $X=Y=0$, and on $\Gamma,(F, G) \not \equiv p(\ell, m)$.

(b) $X, Y \in L^{2}(\Omega) \cap C(\Omega)$ and $X(x, y) \neq 0$ (or $Y(x, y) \neq 0$ ) for $(x, y) \in \Omega$, then there exists at most one pair $((u, v), \Omega)$ with $u, v$ in $C^{1}(\Omega \cup \Gamma) \cap C(\bar{\Omega}) \cap H^{1}(\Omega)$ satisfying (2.3)-(2.6).

Before giving a proof of Theorem 1, we shall first state a result on the convergence of approximate solutions. For this theorem to hold, we need some additional assumptions.

Let the known points of $\gamma$ be $z_{1}=(a, 0), z_{2}=(b, 0),(a<b)$, (cf. Fig. 1). We make the following assumptions

$\left(\mathrm{C}_{1}\right) \Omega$ is convex, $\Gamma$ is the graph of a known piecewise $C^{1}$-function $\varphi:[a, b] \rightarrow \mathbb{R}$ satisfying $\varphi(a)=\varphi(b)=0$ and $\gamma$ is the graph of an unknown convex function $\psi:[a, b] \rightarrow \mathbb{R}$ satisfying $\psi(a)=\psi(b)=0$.

$\left(\mathrm{C}_{2}\right) \psi \in H^{2}(a, b)$ and there is an $M$ such that

$$
\|\psi\|_{H^{2}(a, b)} \leq M .
$$

Let $\Omega_{0}=\left(a_{0}, b_{0}\right) \times\left(a_{0}, b_{0}\right)$ be a known box satisfying $\Omega_{0} \supset \bar{\Omega}$. Let

$$
\mathcal{L} \text { be a compact subset of } H^{1}\left(a_{0}, b_{0}\right) \times H^{1}\left(a_{0}, b_{0}\right) \text {. }
$$


Under the foregoing assumptions, one has

Theorem 2. Let $\left(f_{0}, g_{0}, F_{0}, G_{0}\right) \in\left(H^{2}(\Gamma)\right)^{4}$ satisfy $\left(F_{0}, G_{\Theta}\right) \not \equiv p(\ell, m)$ on $\Gamma$ and assume that the problem (2.3)-(2.6), with the boundary functions $(f, g, F, G)$ replaced by $\left(f_{0}, g_{0}, F_{0}, G_{0}\right)$, has a (unique) solution $\left(\left(u_{0}, v_{0}\right), \Omega\right)$ such that

(i) there is a function $\Phi_{0}(x, y)=(\alpha(y), \beta(x))$ in $\mathcal{L}$ such that, for all $(\xi, \eta) \in \Omega$, we have

$$
\begin{gathered}
\int_{I_{\eta}}\left[u_{0}(x, \eta)-\alpha(\eta)\right] d x=p^{\prime} \cdot\left|I_{\eta}\right| \\
\int_{J_{\xi}}\left[v_{0}(\xi, y)-\beta(\xi)\right] d y=p^{\prime} \cdot\left|J_{\eta}\right|
\end{gathered}
$$

where

$$
I_{\eta}=\Omega \cap\{(x, \eta): x \in \mathbb{R}\} ; \quad J_{\xi}=\Omega \cap\{(\xi, y): y \in \mathbb{R}\}
$$

and $p^{\prime}=\frac{p(1+\nu)(1-2 \nu)}{E}$.

(ii) there is a function $\psi_{0}$ in

$$
K=\left\{\psi \in H^{2}(a, b): \psi \text { is convex and }\|\psi\|_{H^{2}(a, b)} \leq M\right\}
$$

such that $\Omega$ is limited by the graphs of $\varphi, \psi_{0}$.

Then, from any sequence $\left(f_{n}, g_{n}, F_{n}, G_{n}\right)$ satisfying

$$
\left(f_{n}, g_{n}, F_{n}, G_{n}\right) \longrightarrow\left(f_{0}, g_{0}, F_{0}, G_{0}\right) \text { in }\left(L^{2}(\Gamma)\right)^{4},
$$

we can construct a sequence $\left(\psi_{0 n}\right)$ in $K$ such that

$$
\psi_{0 n} \rightarrow \psi_{0} \text { in } C^{1}[a, b] .
$$

\section{Proof of Theorem 1}

The proof is an appropriate modification of the one in [A1]. Let $\left(\left(u^{1}, v^{1}\right), \Omega^{1}\right)$, $\left(\left(u^{2}, v^{2}\right), \Omega^{2}\right)$ satisfying (2.3)-(2.6). We claim that $\Omega^{1}=\Omega^{2},\left(u^{1}, v^{1}\right)=\left(u^{2}, v^{2}\right)$. The proof which is by contradiction, relies on the unique continuation theorem for Lamé systems [A2]. Indeed, suppose by contradiction that $\Omega^{1} \neq \Omega^{2}$. Without loss of generality, we assume that $\Omega^{1} \backslash \Omega^{2} \neq \emptyset$.

Denote by $\omega$ the connected component of $\Omega^{1} \cap \Omega^{2}$ such that $\Gamma \subset \partial \omega$. By uniqueness of continuation for a Lamé system (cf. [A2]), one has

$$
\left(u^{1}, v^{1}\right)=\left(u^{2}, v^{2}\right) \text { in } \bar{\omega}\left(\subset \overline{\Omega^{1} \cap \Omega^{2}}\right) .
$$


Using a result proved in [TA] (Step 3 in Proof of Theorem 1, we can find a connected component $\omega_{0}$ of $\Omega^{1} \backslash \bar{\omega}$ such that $\partial \omega_{0}$ is piecewise $C^{1}$ and that

$$
\partial \omega_{0} \subset(\partial \omega \backslash \Gamma) \cup \gamma^{1}
$$

where $\gamma^{i}(i=1,2)$ is the unknown boundary of $\Omega^{i}$.

Letting $z$ be a point of a smooth arc of $\partial \omega_{0}$, we prove that

$$
\left\{\begin{array}{l}
\ell(z) \sigma_{x}^{1}(z)+m(z) \tau_{x y}^{1}(z)=p \ell(z) \\
m(z) \sigma_{y}^{1}(z)+\ell(z) \tau_{x y}^{1}(z)=p m(z)
\end{array}\right.
$$

where $(\ell(z), m(z))$ is the outer unit normal vector to $\partial \omega_{0}$ at $z$ and $\sigma_{x}^{i}, \sigma_{y}^{i}, \tau_{x y}^{i}$ $(i=1,2)$ are the stress components corresponding to $\left(u^{i}, v^{i}\right)$.

Since

$$
\partial \omega_{0} \subset \partial \omega \subset \partial\left(\Omega^{1} \cap \Omega^{2}\right) \subset \partial \Omega^{1} \cup \partial \Omega^{2}=\Gamma \cup \gamma^{1} \cup \gamma^{2},
$$

two cases are to be considered

(i) $z \in \gamma^{1}$.

(ii) $z \in \partial \omega \cap \gamma^{2}$.

If (i) holds, then $(2,6)$ implies (4.3). If (ii) holds, $(4.1)$ gives

$$
\sigma_{x}^{1}(z)=\sigma_{x}^{2}(z), \quad \sigma_{y}^{1}(z)=\sigma_{y}^{2}(z), \quad \tau_{x y}^{1}(z)=\tau_{x y}^{2}(z) .
$$

But by $(2.6)$,

$$
\left\{\begin{array}{l}
\ell(z) \sigma_{x}^{2}(z)+m(z) \tau_{x y}^{2}(z)=p \ell(z) \\
m(z) \sigma_{y}^{2}(z)+\ell(z) \tau_{x y}^{2}(z)=p m(z)
\end{array}\right.
$$

From (4.4), (4.5) we get (4.3). Hence (4.3) holds for all points $z$ of smooth arcs of $\partial \omega_{0}$.

Now, put

$$
u(x, y)=u^{1}(x, y)-p^{\prime} x ; \quad v(x, y)=v^{1}(x, y)-p^{\prime} y,
$$

where $p^{\prime}=\frac{p(1+\nu)(1-2 \nu)}{E}$. Define $\sigma_{x}, \sigma_{y}, \tau_{x y}$ as in (2.1). In view of (2.3)-(2.6), (4.5), we can verify directly that these functions satisfy (2.3) on $\Omega^{1}$ subject to the conditions

$$
\begin{gathered}
\left.(u, v)\right|_{\Gamma}=\left(f^{\prime}, g^{\prime}\right) \equiv\left(f-p^{\prime} x, g-p^{\prime} y\right) \\
\left\{\begin{array}{l}
\ell \sigma_{x}+m \tau_{x y}=F^{\prime} \\
m \sigma_{y}+\ell \tau_{x y}=G^{\prime}
\end{array} \quad \text { on } \Gamma\right.
\end{gathered}
$$


where $\left(F^{\prime}, G^{\prime}\right)=(F-p \ell, G-p m)$ and

$$
\left\{\begin{array}{l}
\ell \sigma_{x}+m \tau_{x y}=0 \\
m \sigma_{y}+\ell \tau_{x y}=0
\end{array} \quad \text { on } \partial \omega_{0} \cup \gamma^{1}\right.
$$

Using (4.7), (4.8), we shall show that $\left(F^{\prime}, G^{\prime}\right) \equiv(0,0)$ on $\Gamma$ which would contradict (a) in case $X=Y=0$.

Now, multiplying the first equation of (2.3) by $u$, integrating over $\omega_{0}$ and using (4.8), we have

$$
\int_{\omega_{0}}\left(\sigma_{x} \frac{\partial u}{\partial x}+\tau_{x y} \frac{\partial u}{\partial y}\right) d x d y=0 .
$$

Similarly, multiplying the second equation of (2.3) by $v$, integrating over $\omega_{0}$ and using (4.8) we have

$$
\int_{\omega_{0}}\left(\sigma_{y} \frac{\partial v}{\partial y}+\tau_{x y} \frac{\partial v}{\partial x}\right) d x d y=0 .
$$

Substituting the second and the third equations of (2.1) into (4.9), (4.10), adding together the result thus obtained, we get

$$
\int_{\omega_{0}}\left\{\frac{E \nu}{(1+\nu)(1-2 \nu)}\left(\varepsilon_{x}+\varepsilon_{y}\right)^{2}+\frac{E}{1+\nu}\left[\left(\varepsilon_{x}\right)^{2}+\left(\varepsilon_{y}\right)^{2}\right]+G\left(\frac{\partial u}{\partial y}+\frac{\partial v}{\partial x}\right)^{2}\right\} d x d y=0
$$

which gives $\varepsilon_{x}=\varepsilon_{y}=\gamma_{x y}=0$ in $\omega_{0}$, i.e.,

$$
\frac{\partial u}{\partial x}=\frac{\partial v}{\partial y}=\frac{\partial u}{\partial y}+\frac{\partial v}{\partial x}=0
$$

Since $\omega_{0}$ is connected, we can show by elementary technique that there are constants $h, k, k^{\prime}$ satisfying

$$
u(x, y)=h y+k ; \quad v(x, y)=-h x+k^{\prime} \quad \forall(x, y) \in \omega_{0} .
$$

Put $\quad \tilde{u}=u-h y-k, \quad \widetilde{v}=v+h x-k^{\prime}$.

Defining $\widetilde{\sigma}_{x}, \tilde{\sigma}_{y}, \tilde{\tau}_{x y}$ by $(2.1$ ) (with $u, v$ replaced by $\widetilde{u}, \tilde{v}$ ), we can verify directly that

$$
\widetilde{\sigma}_{x}=\sigma_{x}, \quad \widetilde{\sigma}_{y}=\sigma_{y}, \quad \tilde{\tau}_{x y}=\tau_{x y} .
$$

Hence $\widetilde{\sigma}_{x}, \widetilde{\sigma}_{y}, \widetilde{\tau}_{x y}$ satisfy (2.3) with $X=Y=0$ and

$$
\widetilde{u}=\widetilde{v}=0 \text { in } \omega_{0}, \quad \widetilde{\sigma}_{x}=\widetilde{\sigma}_{y}=\widetilde{\tau}_{x y}=0 \text { in } \omega_{0} .
$$

Hence, by uniqueness of continuation for Lamé systems [A2], we obtain $\widetilde{\sigma}_{x}=\widetilde{\sigma}_{y}=$ $\tilde{\tau}_{x y}=0$ in $\Omega^{1}$. This implies in view of $(4.7),(4.11)$ that $\left(F^{\prime}, G^{\prime}\right) \equiv 0$ on $\Gamma$, i.e.,

$$
(F, G) \equiv p(\ell, m) \text { on } \Gamma
$$

a contradiction. 
Finally, let the hypothesis (b) of the theorem hold. Without loss of generality, we can assume that $X(x, y)>0$ for all $(x, y) \in \Omega^{1}$. Integrating the first equation of (2.3) over $\omega_{0}$ and using (4.8) we get

$$
\int_{\omega_{0}} X(x, y) d x d y=0
$$

which contradicts the assumption that $X$ is positive and continuous on $\Omega^{1}$. This completes the proof of Theorem 1 .

\section{Proof of Theorem 2}

We shall construct elements $\psi_{0 n}$ in $K$ as minima of certain functionals. Denoting by $\Omega_{\psi}(\psi$ in $K)$ the domain limited by the known boundary $\Gamma$ and the unknown boundary that is the graph of the function $\psi \in K$. Put

$$
\|(u, v)\|_{\psi}^{2}=\left\|\frac{\partial u}{\partial x}\right\|_{L^{2}\left(\Omega_{\psi}\right)}^{2}+\left\|\frac{\partial v}{\partial y}\right\|_{L^{2}\left(\Omega_{\psi}\right)}^{2}+\left\|\frac{\partial u}{\partial y}+\frac{\partial v}{\partial x}\right\|_{L^{2}\left(\Omega_{\psi}\right)}^{2} .
$$

We can verify directly that $\|\cdot\|_{\psi}$ is a norm on the space

$$
H_{\Gamma}^{1}\left(\Omega_{\psi}\right)=\left\{(u, v) \in\left(H^{1}\left(\Omega_{\psi}\right)\right)^{2}: \int_{I_{\eta}} u(x, \eta) d x=0, \int_{J_{\xi}} v(\xi, y) d y=0 \text { for }(\xi, \eta) \in \Omega\right\}
$$

and that $\quad V\left(\Omega_{\psi}\right)=$ the completion of $H_{\Gamma}^{1}\left(\Omega_{\psi}\right)$ with respect to the norm $\|\cdot\|_{\psi}$ is a Hilbert space.

The proof will be divided into two steps. In Step 1, we shall transform (2.3)-(2.6) into a variational problem on $V\left(\Omega_{\psi}\right)$ and construct the $\left(\psi_{0 n}\right)$ 's as minima of certain functionals. In Step 2, we shall prove that $\psi_{0 n} \rightarrow \psi_{0}$ in $C^{1}[a, b]$.

Step 1. Transformation of (2.3)-(2.6) into a variational problem on $V\left(\Omega_{\psi}\right)$ and construction of the $\left(\psi_{0 n}\right)$ 's.

We set some notations

$$
\begin{aligned}
U & =\left(u^{\prime}, v^{\prime}\right)=\left(u-p^{\prime} x, v-p^{\prime} y\right), \\
W & =\left(u_{1}, v_{1}\right) \\
\left(F^{\prime}, G^{\prime}\right) & =(F-p \ell, G-p m), \\
\left(f_{0}^{\prime}, g_{0}^{\prime}\right) & =\left(f_{0}-p^{\prime} x, g_{0}-p^{\prime} y\right) \\
\left(f_{n}^{\prime}, g_{n}^{\prime}\right) & =\left(f_{n}-p^{\prime} x, g_{n}-p^{\prime} y\right)
\end{aligned}
$$

For any $\left(u_{1}, v_{1}\right)=W \in V\left(\Omega_{\psi}\right)$, multiplying (2.3) by $u_{1}, v_{1}$ successively, adding the results thus obtained and using the divergence theorem, we get, in view of (4.6)-(4.8), that 


$$
a_{\psi}(U, W)=L_{F, G}(W) \quad \forall W \in V\left(\Omega_{\psi}\right)
$$

where

$$
\begin{aligned}
L_{F, G}(W)= & \int_{\Gamma}\left(u_{1} F^{\prime}+v_{1} G^{\prime}\right) d s \\
a_{\psi}(U, W)= & \int_{\Omega_{\psi}}\left\{\frac{E \nu}{(1+\nu)(1-2 \nu)}\left(\frac{\partial u^{\prime}}{\partial x}+\frac{\partial v^{\prime}}{\partial y}\right)\left(\frac{\partial u_{1}}{\partial x}+\frac{\partial v_{1}}{\partial y}\right)+\right. \\
& \left.+\frac{E}{1+\nu}\left(\frac{\partial u^{\prime}}{\partial x} \frac{\partial u_{1}}{\partial x}+\frac{\partial v^{\prime}}{\partial y} \frac{\partial v_{1}}{\partial y}\right)+G\left(\frac{\partial u^{\prime}}{\partial y}+\frac{\partial v^{\prime}}{\partial x}\right)\left(\frac{\partial u_{1}}{\partial y}+\frac{\partial v_{1}}{\partial x}\right)\right\} d x d y .
\end{aligned}
$$

From the above argument it follows that the problem (2.3)-(2.6) is equivalent to the problem of finding a pair $(U, W)$ satisfying $(5.1)$ and

$$
\left.U\right|_{\Gamma}=\left(f_{0}^{\prime}, g_{0}^{\prime}\right)\left(\equiv\left(f_{0}-p^{\prime} x, g_{0}-p^{\prime} y\right)\right) .
$$

We shall apply the Lax-Milgram theorem to the latter problem. To this end, we shall decompose $U$ into a sum of a function $Y$ in $V\left(\Omega_{\psi}\right)$ and a $\Phi \in \mathcal{L}$, i.e.,

$$
U=Y+\Phi
$$

Using (5.3) we can rewrite (5.1) as follows

$$
a_{\psi}(Y, W)=-a_{\psi}(\Phi, W)+L_{F, G}(W) \quad \forall W \in V\left(\Omega_{\psi}\right) .
$$

Thus, our problem is equivalent to that of finding a triplet $(Y, \Phi, \psi)$ satisfying (5.4) and $\left.\quad Y\right|_{\Gamma}=-\left.\Phi\right|_{\Gamma}+\left(f_{0}^{\prime}, g_{0}^{\prime}\right)$.

We claim that, for each $(\Phi, \psi, F, G)$ in $\mathcal{L} \times K \times\left(L^{2}(\Gamma)\right)^{2}$, the problem (5.4) has a unique solution $Y(\cdot ; \Phi, \psi, F, G)$ in $V\left(\Omega_{\psi}\right)$. Indeed, $a_{\psi}$ is coercive in $V\left(\Omega_{\psi}\right)$. We verify that $L_{F, G}$ is continuous on $V\left(\Omega_{\psi}\right)$. One has by direct computations

$$
\left|L_{F, G}\left(u_{1}, v_{1}\right)\right| \leq\left\|F^{\prime}\right\|_{L^{2}(\Gamma)}\left\|u_{1}\right\|_{L^{2}(\Gamma)}+\left\|G^{\prime}\right\|_{L^{2}(\Gamma)}\left\|v_{1}\right\|_{L^{2}(\Gamma)} .
$$

Let $(\xi, \eta) \in \Gamma$. Since $\left(u_{1}, v_{1}\right) \in V\left(\Omega_{\psi}\right)$ one has

$$
\int_{J_{\xi}} v_{1}(\xi, \eta) d y=0 .
$$

Hence

$$
\begin{aligned}
v_{1}(\xi, \eta) & =v_{1}(\xi, \eta)-\frac{1}{\left|J_{\xi}\right|} \int_{J_{\xi}} v_{1}(\xi, y) d y=\frac{1}{\left|J_{\xi}\right|} \int_{J_{\xi}}\left[v_{1}(\xi, \eta)-v_{1}(\xi, y)\right] d y \\
& =\frac{1}{\left|J_{\xi}\right|} \int_{J_{\xi}} \int_{y}^{\eta} \frac{\partial v_{1}}{\partial y}(\xi, \tau) d \tau d y .
\end{aligned}
$$


It follows that there is a constant $C>0$ satisfying

$$
\left\|v_{1}\right\|_{L^{2}(\Gamma)}^{2} \leq C\left\|\frac{\partial v_{1}}{\partial y}\right\|_{L^{2}\left(\Omega_{\psi}\right)}^{2} \leq\left\|\left(u_{1}, v_{1}\right)\right\|_{\psi^{*}}^{2}
$$

Similarly,

$$
\left\|u_{1}\right\|_{L^{2}(\Gamma)}^{2} \leq C\left\|\left(u_{1}, v_{1}\right)\right\|_{\psi}^{2} .
$$

Substituting (5.6), (5.7) into (5.5) gives that $L_{F, G}$ is a continuous linear functional on $V\left(\Omega_{\psi}\right)$.

By using the Lax-Milgram theorem, there exists, for each $(\Phi, \psi, F, G)$ in $\mathcal{L} \times K \times\left(L^{2}(\Gamma)\right)^{2}$, a unique solution $Y(\cdot ; \Phi, \psi, F, G)$ in $V\left(\Omega_{\psi}\right)$ of equation (5.4). The problem becomes one of finding a $(\Phi, \psi)$ in $\mathcal{L} \times K$ such that

$$
\left.Y\left(\cdot ; \Phi, \psi, F_{0}, G_{0}\right)\right|_{\Gamma}=-\left.\Phi\right|_{\Gamma}+\left(f_{0}^{\prime}, g_{0}^{\prime}\right)
$$

Since $\left(f_{n}, g_{n}, F_{n}, G_{n}\right)$ is in $\left(L^{2}(\Gamma)\right)^{4}$, we can pose

$$
J_{n}(\Phi, \psi)=\int_{\Gamma}\left|Y\left(x, y ; \Phi, \psi, F_{n}, G_{n}\right)+\Phi(x, y)-\left(f_{n}^{\prime}(x, y), g_{n}^{\prime}(x, y)\right)\right|^{2} d s
$$

where, we recall, $\left(f_{n}^{\prime}, g_{n}^{\prime}\right)=\left(f_{n}-p^{\prime} x, g_{n}-p^{\prime} y\right)$.

Now, consider the problem

$$
\text { Find } \min \left\{J_{n}(\Phi, \psi): \forall(\Phi, \psi) \in \mathcal{L} \times K\right\} .
$$

The existence of a solution of (5.9) is a direct consequence of the following lemma (the proof of which will be given later).

Lemma 1. Let $\left(\Phi_{m}, \psi_{m}\right)$ is a sequence in $\mathcal{L} \times K$. Then there exists a $(\Phi, \psi)$ in $\mathcal{L} \times K$ and a subsequence $\left(\Phi_{m_{k}}, \psi_{m_{k}}\right)$ such that

$$
\begin{array}{ll}
\Phi_{m_{k}} \rightarrow \Phi & \text { in }\left(H^{1}\left(a_{0}, b_{0}\right)\right)^{2} \\
\psi_{m_{k}} \rightarrow \psi & \text { in } H^{2}(a, b)
\end{array}
$$

and that

$$
\begin{array}{r}
\left.Y\left(\cdot ; \Phi_{m_{k}}, \psi_{m_{k}}, F_{n}, G_{n}\right)\right|_{\Gamma} \rightarrow Y\left(\cdot ; \Phi, \psi, F_{n}, G_{n}\right) \\
\left.Y\left(\cdot ; \Phi_{m_{k}}, \psi_{m_{k}}, F_{m_{k}}, G_{m_{k}}\right)\right|_{\Gamma} \rightarrow Y\left(\cdot ; \Phi, \psi, F_{0}, G_{0}\right)
\end{array}
$$

in $\left(L^{2}(\Gamma)\right)^{2}$ as $k \rightarrow \infty$.

Now, we consider the existence problem for (5.9). Put

$$
\theta=\inf \left\{J_{n}(\Phi, \psi): \forall(\Phi, \psi) \in \mathcal{L} \times K\right\} .
$$


For each $m$, there exists a pair $\left(\Phi_{m}, \psi_{m}\right) \in \mathcal{L} \times K$ such that

$$
\theta \leq J_{n}\left(\Phi_{m}, \psi_{m}\right)<\theta+\frac{1}{m} .
$$

By the compactness of $\mathcal{L} \times K$, there exist a $\left(\Phi_{0 n}, \psi_{0 n}\right) \in \mathcal{L} \times K$ and a subsequence $\left(\Phi_{m_{k}}, \psi_{m_{k}}\right)$ in $\mathcal{L} \times K$ such that

$$
\begin{array}{ll}
\Phi_{m_{k}} \rightarrow \Phi_{0 n} & \text { in }\left(H^{1}\left(a_{0}, b_{0}\right)\right)^{2} \\
\psi_{m_{k}} \rightarrow \psi_{0 n} & \text { in } H^{2}(a, b)
\end{array}
$$

From (5.12) one has

$$
\theta \leq J_{n}\left(\Phi_{0 n}, \psi_{0 n}\right) \leq \liminf _{k \rightarrow \infty} J_{n}\left(\Phi_{m_{k}}, \psi_{m_{k}}\right) \leq \theta .
$$

Hence $J_{n}\left(\Phi_{0 n}, \psi_{0 n}\right)=\theta$, i.e., $\left(\Phi_{0 n}, \psi_{0 n}\right)$ is a solution of (5.9).

Step 2. Convergence of $\left(\psi_{0 n}\right)$ to $\psi_{0}$.

We have in view of the decomposition

$$
\left(u_{0}^{\prime}, v_{0}^{\prime}\right)=Y\left(\cdot ; \Phi_{0}, \psi_{0}, F_{0}, G_{0}\right)+\Phi_{0}
$$

where $\left(u_{0}^{\prime}, v_{0}^{\prime}\right)=\left(u_{0}-p^{\prime} x, v_{0}-p^{\prime} y\right)$, that

$$
\begin{aligned}
J_{n}\left(\Phi_{0}, \psi_{0}\right) & =\int_{\Gamma_{0}}\left|Y\left(x, y ; \Phi_{0}, \psi_{0}, F_{0}, G_{0}\right)+\Phi_{0}(x, y)-\left(f_{n}^{\prime}, g_{n}^{\prime}\right)\right|^{2} d s \\
& =\int_{\Gamma_{0}}\left|\left(f_{0}-f_{n}, g_{0}-g_{n}\right)\right|^{2} d s .
\end{aligned}
$$

Hence

$$
J_{n}\left(\Phi_{0}, \psi_{0}\right) \rightarrow 0 \text { as } n \rightarrow \infty .
$$

By the definition of $\left(\Phi_{0 n}, \psi_{0 n}\right)$, one has

$$
0 \leq J_{n}\left(\Phi_{0 n}, \psi_{0 n}\right) \leq J_{n}\left(\Phi_{0}, \psi_{0}\right) .
$$

From (5.16), the latter inequalities imply

$$
\lim _{n \rightarrow \infty} J_{n}\left(\Phi_{0 n}, \psi_{0 n}\right)=0 .
$$

By the compactness of $K$, we can find a subsequence $\left(\psi_{0 n_{k}}\right)$ of $\left(\psi_{0 n}\right)$ and a $\psi \in K$ such that

$$
\psi_{0 n_{k}} \rightarrow \psi \quad \text { in } H^{2}(a, b) .
$$


It is sufficient to prove that

$$
\psi=\psi_{0}
$$

which would imply that

$$
\psi_{0 n} \rightarrow \psi_{0} \text { in } H^{2}(a, b)
$$

as desired.

By the compactness of $\mathcal{L} \times K$, there are a subsequence of $\left(\Phi_{0 n_{k}}, \psi_{0 n_{k}}\right)$ (still represented by the same notation) and a $\Phi$ in $\mathcal{L}$ such that

$$
\Phi_{0 n_{k}} \rightarrow \Phi \quad \text { in }\left(H^{1}\left(a_{0}, b_{0}\right)\right)^{2} .
$$

In view of (5.18), (5.20), Lemma 1 implies

$$
\left.\left.Y\left(\cdot ; \Phi_{0 n_{k}}, \psi_{0 n_{k}}, F_{n_{k}}, G_{n_{k}}\right)\right|_{\Gamma} \rightarrow Y\left(\cdot ; \Phi, \psi, F_{0}, G_{0}\right)\right|_{\Gamma} \text { in }\left(L^{2}(\Gamma)\right)^{2} .
$$

Hence, one has in view of (5.17) that

$$
\int_{\Gamma}\left|Y\left(\cdot ; \Phi, \psi, F_{0}, G_{0}\right)+\Phi-\left(f_{0}^{\prime}, g_{0}^{\prime}\right)\right|^{2} d s \leq \liminf _{k \rightarrow \infty} J_{n_{k}}\left(\Phi_{0 n_{k}}, \psi_{0 n_{k}}\right)=0 .
$$

Thus $(Y, \Phi, \psi)$ satisfies (5.8). It follows that $(U, \psi)=(Y+\Phi, \psi)$ satisfies (5.1) and (5.2).

Now, we prove that $(U, \psi)=\left(\left(u_{0}^{\prime}, v_{0}^{\prime}\right), \psi_{0}\right)$. The proof relies on a result of $[\mathrm{F}]$. We first verify the regularity of $U$. Assume that $U=\left(\bar{u}^{\prime}, \bar{v}^{\prime}\right)$. Put $\bar{e}=\partial \bar{u}^{\prime} / \partial x+\partial \bar{v}^{\prime} / \partial y$. Then one get from (5.1) that

$$
\Delta \bar{e}=0
$$

in the sense of distribution. Since $\bar{e} \in L^{2}\left(\Omega_{\psi}\right)$, it follows that $\bar{e} \in C^{\infty}\left(\Omega_{\psi}\right)$ (see, e.g., $[\mathrm{F}]$, page 56$)$. We also have in view of $(2.1)$

$$
\Delta \bar{u}^{\prime}=-\frac{2 \nu}{1-\nu} \frac{\partial \bar{e}}{\partial x}, \quad \Delta \bar{v}^{\prime}=-\frac{2 \nu}{1-\nu} \frac{\partial \bar{e}}{\partial y}
$$

in the sense of distribution.

In the same way as for $(5.6),(5.7)$, we have

$$
\left\|\bar{u}^{\prime}\right\|_{L^{2}\left(\Omega_{\psi}\right)} \leq C\left\|\frac{\partial \bar{u}^{\prime}}{\partial x}\right\|_{L^{2}\left(\Omega_{\psi}\right)^{\prime}},\left\|\bar{v}^{\prime}\right\|_{L^{2}\left(\Omega_{\psi}\right)} \leq C\left\|\frac{\partial \bar{v}^{\prime}}{\partial y}\right\|_{L^{2}\left(\Omega_{\psi}\right)}
$$

It follows that $\bar{u}^{\prime}, \bar{v}^{\prime}$ are in $L^{2}\left(\Omega_{\psi}\right)$. Hence, using the regularity results (see $[\mathrm{F}]$, page 56) one gets that $\bar{u}^{\prime}, \bar{v}^{\prime} \in C^{\infty}\left(\Omega_{\psi}\right)$. 
From the equality

$$
\bar{v}^{\prime}(\xi, \eta)=\bar{v}^{\prime}(\xi, 0)+\int_{0}^{\eta} \frac{\partial \bar{v}^{\prime}}{\partial y}(\xi, y) d y \quad(\xi, \eta) \in \Omega_{\psi}
$$

one gets $\bar{v}^{\prime} \in C\left(\Omega_{\psi} \cup \Gamma\right)$. Similarly $\bar{u}^{\prime} \in C\left(\Omega_{\psi} \cup \Gamma\right)$. By the smoothness assumption on $\left(f_{0}, g_{0}, F_{0}, G_{0}\right)$, we have that $\bar{u}^{\prime}, \bar{v}^{\prime} \in C^{1}\left(\Omega_{\psi} \cup \Gamma\right)$. It follows that $\left(\bar{u}^{\prime}, \bar{v}^{\prime}\right)$ satisfies the conditions of Theorem 1. By the uniqueness result in the latter theorem, it follows that

$$
((\bar{u}, \bar{v}), \psi)=\left(\left(u_{0}, v_{0}\right), \psi_{0}\right)
$$

where $(\bar{u}, \bar{v})=\left(\bar{u}^{\prime}+p^{\prime} x, \bar{v}^{\prime}+p^{\prime} y\right)$. This will complete the proof of Theorem 2 once Lemma 1 is proved.

Proof of Lemma 1. Since $\mathcal{L}$ is compact in $H^{1}\left(\Omega_{0}\right)$ and since $\left(\psi_{m}\right)$ is bounded in $H^{2}(a, b)$, there exists a subsequence $\left(\Phi_{m_{k}}, \psi_{m_{k}}\right)$ of $\left(\Phi_{m}, \psi_{m}\right)$ such that $(5.10),(5.11)$ hold. We next prove that (5.12), (5.13) hold. We prove (5.13). The proof of (5.12) which is similar is omitted. For notational convenience, we put

$$
\begin{aligned}
Z_{m} & =Y\left(\cdot ; \Phi_{m}, \psi_{m}, F_{m}, G_{m}\right) \\
Z_{0} & =Y\left(\cdot ; \Phi, \psi, F_{0}, G_{0}\right) \\
\widetilde{\psi}_{m} & =\max \left\{\psi_{m}, \psi\right\} .
\end{aligned}
$$

From (5.4)-(5.7), we can find a constant $M$ such that

$$
\left\|Z_{m}\right\|_{V\left(\Omega_{\tilde{\psi}}\right)} \leq M \text { for } m=0,1,2, \ldots
$$

For $W$ in $H^{1}\left(\Omega_{0}\right)$, we get by direct computation,

$$
\begin{aligned}
\left|a_{\tilde{\psi}_{m}}\left(Z_{m}-Z_{0}, W\right)\right| \leq & \left|a_{\psi_{m}}\left(Z_{m}, W\right)-a_{\psi}\left(Z_{0}, W\right)\right|+ \\
& +C\left\|Z_{m}\right\|_{V\left(\Omega_{\psi_{m}}\right)}\|W\|_{L^{2}\left(\Omega_{\psi_{m}} \backslash \Omega_{\psi}\right)} \\
& +C\left\|Z_{0}\right\|_{V\left(\Omega_{\psi_{m}}\right)}\|W\|_{L^{2}\left(\Omega_{\psi} \backslash \Omega_{\psi_{m}}\right)} \\
\leq & \left|a_{\psi_{m}}\left(\Phi_{m}, W\right)-a_{\psi_{m}}(\Phi, W)\right|+ \\
& +C\left(\left\|F_{0}-F_{m}\right\|_{L^{2}(\Gamma)}+\left\|G_{0}-G_{m}\right\|_{L^{2}(\Gamma)}\right) \\
& +C M\left(\|W\|_{L^{2}\left(\Omega_{\psi_{m}} \backslash \Omega_{\psi}\right)}+\|W\|_{L^{2}\left(\Omega_{\psi} \backslash \Omega_{\psi_{m}}\right)}\right) .
\end{aligned}
$$

In view of (5.4), (5.5), the latter inequality implies that

$$
\left|a_{\tilde{\psi}_{m}}\left(Z_{m}-Z_{0}, W\right)\right| \rightarrow 0 \text { as } m \rightarrow \infty
$$

In view of the representation (5.21), it follows from (5.23) that

$$
\left.\left.Z_{m}\right|_{\Gamma_{0}} \rightarrow Z_{0}\right|_{\Gamma_{0}} \text { in }\left(L^{2}\left(\Gamma_{0}\right)\right)^{2} .
$$

This proves Lemma 1 and hence the proof of Theorem 2 is completed. 


\section{Acknowledgements}

The authors wish to thank the referee for his most interesting remarks and constructive criticisms.

Financially supported by the Vietnam Program of Basic Research in the Natural Sciences.

\section{REFERENCES}

[A1] D. D Ang, D. D. Trong and Yamamoto M. Unique continuation and identification of boundary of an elastic body, Journal of Inverse and Ill-posed Problem 3, $n^{\circ} 6,1996,417-428$.

[A2] D. D. Ang, Ikehata M., D. D. Trong and Yamamoto M. Unique continuation for a stationary isotropic Lamé System with variable coefficients, Commun. in Partial Diff. Eqs. 23, 1998, 371-385.

[F] Friedman A. Partial Differential Equations, Holt Rinehart and Winston Inc., 1969.

[TA] D. D. Trong and D. D. Ang. Domain identification for rlliptic equations: the Zero Flux Case, Z. Anal. Anwen. 19, No 1 (2000), pp 109-120.

[TG] Timosenko S. P. and Goodier J. N. Theory of Elasticity, Mc Graw-Hill, New York, 1970.

Received June 17, 2001

in revised form January 12, 2002

\section{XÁC ĐỊNH BIÊN CƯA MỘT VẠT THỄ ĐÀN HỒI NHÚNG MỘT PHẦN TRONG CHẤT LỎNG}

Xét một trụ đàn hồi dài có tiết diện không đởi $\Omega$ được nhúng một phần trong một chất lơng có mật độ không đổi. Giả thiết rằng vật thể ở trạng thái biến dạng phẳng và $\Omega$ có biên gồm hai cung trơn từng đoạn gặp nhau tại hai điểm trên đường thẳng nằm ngang. Biên trên $\Gamma$ được biết trước, biên dưới $\gamma$ hoàn toàn nằm trong chất lơng, là chưa biết và phải xác định. Giả thiết biết trường chuyển vị và ứng suất mặt trên một phần của $\Gamma$, và biên dưới chịu tác dụng của áp suất thủy tĩnh không đổi, các tác giả chứng minh một định lý về tính dủy nhất, và trong trường hợp tồn tại nghiệm, sẽ chỉ ra sự tồn tại của một dãy các nghiệm chính quy hóa hội tụ về nghiệm, sẽ chỉ ra sự tồn tại của một dãy các nghiệm chính quy hóa hội tụ về nghiệm chính xác. 